\title{
ALMOST HERMITIAN SUBMERSIONS
}

\author{
BILL WATSON
}

\section{Introduction}

In this article, we examine the differential geometric properties of almost complex Riemannian submersions between almost Hermitian manifolds (almost Hermitian submersions). In $\S 2$ we define the principal objects of our study after recalling in $\S 1$ the definitions of various almost Hermitian structures. In $\S 3$ we prove one of our main theorems:

3.1. Theorem. Let $f: M \rightarrow B$ be an almost Hermitian submersion with $M$, an almost semi-Kähler manifold. Then $B$ is an almost semi-Kähler manifold if and only if the fibres $F_{y}$ of $f$ are minimal submanifolds of $M$.

In $\S 4$ we prove that the horizontal distribution of an almost Hermitian submersion whose total space is Kähler is completely integrable. We thereby extend a result of Ako [1, Th. 5.1, p. 502], who studied projectable tensor fields on fibre spaces with almost complex structures. We collect Ako's result as Corollary 4.1.3.

The relations between the holomorphic sectional and bisectional curvatures of the two manifolds of an almost Hermitian submersion are studied in $\S 5$. Surprisingly, an almost Hermitian submersion whose total space is quasi-Kähler preserves holomorphic sectional curvature on horizonal vectors and decreases the holomorphic bisectional curvature on pairs of horizontal vectors in contrast to the well-known increasing effect of Riemannian submersions on the Riemannian sectional curvature. $\S 6$ extends the results on Betti numbers in [19] to inequalities on the numbers $b_{1,0}$ and $b_{0,1}$ of linearly independent harmonic 1 -forms of bidegrees $(1,0)$ and $(0,1)$ respectively. In particular, we find

6.5. Theorem. Let $M$ and $B$ be compact almost semi-Kähler manifolds. If there exists an almost Hermitian submersion $f: M \rightarrow B$, then

$$
b_{1,0}(B) \leq b_{1,0}(M), \quad b_{0,1}(B) \leq b_{0,1}(M) .
$$

Finally in $\S 7$ we show that almost quaternionic submersions between $q$ quasi-Kähler manifolds are locally product mappings.

The author would like to express his gratitude to the referee for his comments on the original manuscript and for his communication of the unpublished results of A. M. Naveira and L. Hervella, who showed that our previously

Received August 21, 1973, and, in, revised form, October 25, 1974. 
announced class of para-Kähler manifolds actually coincides with the known class of quasi-Kähler manifolds.

The author acknowledges the partial support of a Beca de Investigación from La Universidad de Oriente, Cumaná, Venezuela.

\section{Almost Hermitian structures and submanifolds}

An almost complex structure on a smooth manifold $M$ is a smooth tensor field $J$ of type $(1,1)$ such that $J^{2}=-$ identity. An almost complex manifold is such a manifold with a chosen almost complex structure. An almost complex manifold is necessarily orientable and must have an even dimension. An almost Hermitian manifold is an almost complex manifold $(M, J)$ with a chosen Riemannian structure $g$ which satisfies

$$
g(X, Y)=g(J X, J Y)
$$

for all $X, Y \in \mathscr{D}(M)$, the Lie algebra of smooth vector fields on $M$. All manifolds considered in this article are smooth, connected, complete Riemannian manifolds. Additional structures will be fully described when they are invoked.

The Kähler form of an almost Hermitian manifold $(M, g, J)$ is the smooth differential 2-form of bidegree $(1,1)$ given by

$$
\Phi(X, Y)=g(X, J Y),
$$

for all $X, Y \in \mathscr{D}(M)$. The Levi-Civita connection $V$ of the almost Hermitian manifold $M$ can be extended to the full tensor algebra on $M$, and in this manner we have tensor fields such as $\nabla_{X} J$, etc. In fact,

$$
\begin{aligned}
\nabla_{X} J(Y) & =\nabla_{X}(J Y)-J \nabla_{X} Y, \\
\nabla_{X} \Phi(Y, Z) & =g\left(\nabla_{X} J(Y), Z\right) \\
d \Phi(X, Y, Z) & =\nabla_{X} \Phi(Y, Z)+\nabla_{Y} \Phi(Z, X)+\nabla_{Z} \Phi(X, Y), \\
\delta \Phi(X) & =-\sum_{i=1}^{m}\left\{\nabla_{E_{i}} \Phi\left(E_{i}, X\right)+\nabla_{J E_{i}} \Phi\left(J E_{i}, X\right)\right\},
\end{aligned}
$$

where $\left\{E_{1}, \cdots, E_{m}, J E_{1}, \cdots, J E_{m}\right\}$ is a local basis for $\mathscr{D}(U)$ on an open subset $U$ of $\left(M^{2 m}, g, J\right)$.

The Nijenhuis tensor of the almost complex structure $J$ is the tensor field $N$ of type $(1,2)$ given by

$$
N(X, Y)=[X, Y]+J[J X, Y]+J[X, J Y]-[J X, J Y] .
$$

A well-known theorem of Newlander-Nirenberg states that $J$ is the almost complex structure associated to a complex manifold structure on $M$ if and only if the Nijenhuis tensor of $J$ vanishes, in which case we say that $J$ is integrable. 
We recall the definitions of some of the well-known classes of almost Hermitian manifolds [7], [14]. Let $\mathscr{A} \mathscr{H}$ denote the class of almost Hermitian manifolds. Then $(M, g, J) \in \mathscr{A} \mathscr{H}$ is said to be :

(a) $\operatorname{Kähler}(\mathscr{K})$ if $\nabla_{X} J=0$,

(b) almost Kähler $(\mathscr{A} \mathscr{K})$ if $d \Phi=0$,

(c) almost Tachibana $(\mathscr{A} \mathscr{T})$ if $\nabla_{X} J(X)=0$,

(d) quasi-Kähler $(\mathscr{Q} \mathscr{K})$ if $d \Phi^{(2,1)}=d \Phi^{(1,2)}=0$,

(e) almost semi-Kähler $(\mathscr{A} \mathscr{S} \mathscr{K})$ if $\delta \Phi=0$,

(f) Hermitian $(\mathscr{H})$ if $N=0$,

(g) $\operatorname{semi-Kähler}(\mathscr{S} \mathscr{K})$ if $\delta \Phi=0$ and $N=0$.

Remark. The geometry of almost Tachibana spaces have been extensively examined in [10] where they are called nearly Kähler spaces. Wolf and Gray [22] studied the almost Hermitian structures on certain homogeneous spaces. Examples of each class may be found in [8]. Finally, we note that it is easy to show that $\mathscr{K}=\mathscr{A} \mathscr{K} \cap \mathscr{A} \mathscr{T}=\mathscr{Q} \mathscr{K} \cap \mathscr{H}$.

These classes of almost Hermitian manifolds are related by the following lattice in which each inclusion is strict:

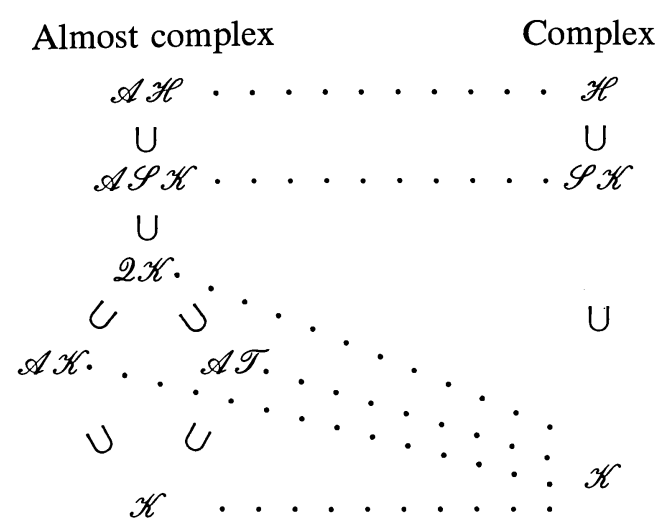

Fig. 1. Lattice of almost Hermitian structures

A manifold mapping $f:(M, J) \rightarrow\left(N, J^{\prime}\right)$ is said to be almost complex if it commutes with the almost complex structures; that is, if $f_{*} J=J^{\prime} f_{*}$. An almost complex mapping between complex manifolds is said to be holomorphic. Almost complex mappings preserve the bidegree of differential forms.

Let $S$ be an immersed submanifold of a Riemannian manifold $M$, and give $S$ the inherited Riemannian structure from $M$. Let $\overline{\mathscr{D}}(S)$ denote the Lie algebra of smooth vector fields on $M$, restricted to the submanifold $S$. Let $\mathscr{D}(S)$ be the subspace of vector fields tangent to $S$, and $\mathscr{D}(S)^{\perp}$ the vector fields orthogonal to $S$. Then we have a direct sum decomposition: $\overline{\mathscr{D}}(S)=\mathscr{D}(S) \oplus \mathscr{D}(S)^{\perp}$. We denote the tensors associated to the submanifold $S$ by a caret ${ }^{~}$. For instance, $\nabla$ denotes the Riemannian connection of $M$ acting on $\overline{\mathscr{D}}(S)$, while $\hat{\nabla}$ is the 
Riemannian connection for $\mathscr{D}(S)$ arising from the induced Riemannian structure on $S$.

The configuration tensor [7] of the immersion of the submanifold $S$ is the tensor $\hat{T}: \mathscr{D}(S) \times \overline{\mathscr{D}}(S) \rightarrow \overline{\mathscr{D}}(S)$ given by

(1) $\hat{T}_{X} Y=\nabla_{X} Y-\hat{\nabla}_{X} Y$, when $X, Y \in \mathscr{D}(S)$,

(2) $\hat{T}_{X} Z=\pi\left(\nabla_{X} Z\right)$, when $X \in \mathscr{D}(S), Z \in \mathscr{D}(S)^{\perp}$, and $\pi: \overline{\mathscr{D}}(S) \rightarrow \mathscr{D}(S)$ is the orthogonal projection mapping.

We collect a few important properties of the configuration tensor $\hat{T}$ in

1.1. Proposition. Let $S$ be an immersed submanifold of the Riemannian manifold $M$. For $X, Y \in \mathscr{D}(S)$, we have

(i) $\hat{T}_{X}$ reverses the subspaces $\mathscr{D}(S)$ and $\mathscr{D}(S)^{\perp}$,

(ii) $\hat{T}_{X} Y=\hat{T}_{Y} X$,

(iii) $\hat{T}_{X}$ is a skew-symmetric linear operator,

(iv) $\pi(R(X, Y))=\hat{R}(X, Y)-\left[\hat{T}_{X}, \hat{T}_{Y}\right]$, (Gauss equation).

Proof. [7].

The mean curvature vector field of the submanifold $S^{n}$ of the Riemannian manifold $M_{m}$ is defined by

$$
H=\sum_{i=1}^{n} \hat{T}_{E_{i}} E_{i}
$$

where $\left\{E_{1}, \cdots, E_{n}\right\}$ is a local orthonormal basis for $\mathscr{D}(S) . S$ is said to be a minimal submanifold of $M$ if $H=0$, and a totally geodesic submanifold of $M$ if $\hat{T}=0$. Obviously, totally geodesic submanifolds are minimal.

An almost Hermitian manifold $S$ immersed in an almost Hermitian manifold $M$ is called an almost Hermitian submanifold of $M$ if the almost complex structure $\hat{J}$ on $S$ is identical to the restriction of the almost complex structure $J$ of $M$. When there is no danger of confusion, we shall denote both almost complex structures by $J$.

We collect from Gray [7] the results on almost Hermitian submanifolds which we shall need in the sequel.

1.2. Proposition. Let $S$ be an almost Hermitian submanifold of an almost Hermitian manifold $M$. If
$M$ is $\left\{\begin{array}{l}\text { (b) almost Kähler, } \\ \text { (c) almost Tachibana, then } S \text { is }\end{array}\right.$
(a) quasi-Kähler,
(d) Kähler,
(e) Hermitian,
(a) quasi-Kähler,
(b) almost Kähler,
(c) almost Tachibana,
(d) Kähler,
(e) Hermitian.

1.3. Proposition. Let $S$ be an almost Hermitian submanifold of the quasiKähler manifold $M$. Then, for all $X, Y \in \mathscr{D}(S)$,

$$
\hat{T}_{X} Y+\hat{T}_{J X} J Y=0 .
$$

In addition to the classical coderivative $\delta \Phi$ of the Kähler form $\Phi$ on the 
ambient almost Hermitian manifold $M$, it is possible to define another tensor, called the partial coderivative of $\Phi$ with respect to the almost Hermitian submanifold $S$, and denoted $\tilde{\delta} \Phi$. Letting $\left\{E_{1}, \cdots, E_{n}, J E_{1}, \cdots, J E_{n}\right\}$ be a local $J$-basis for $\mathscr{D}(S)$, Gray defined $\tilde{\delta} \Phi(X)$ for any $X \in \overline{\mathscr{D}}(S)$ via

$$
\tilde{\delta} \Phi(X)=-\sum_{i=1}^{n}\left\{\nabla_{E_{i}} \Phi\left(E_{i}, X\right)+\nabla_{J E_{i}} \Phi\left(J E_{i}, X\right)\right\},
$$

and then was able to show

1.4. Theorem. Let $S$ be an almost Hermitian submanifold of an almost Hermitian manifold $M$. Then $S$ is a minimal submanifold of $M$ if and only if $\tilde{\delta} \Phi(Z)=0$ for all $Z \in \mathscr{D}(S)^{\perp}$.

A consequence of this theorem and Proposition 1.3 is

1.5. Theorem. Every almost Hermitian submanifold of a quasi-Kähler manifold is minimally immersed.

\section{Almost Hermitian submersions}

Almost Hermitian submersions are a natural generalization of almost complex product projection mappings and almost complex covering mappings. We propose to present in this section a globally invariant formulation of some of the properties of these interesting mappings.

Let $\left(M^{2 m}, g, J\right)$ and $\left(B^{2 n}, h, J^{\prime}\right)$ be almost Hermitian manifolds. A smooth surjective mapping $f: M \rightarrow B$ is called a Riemannian submersion [15] if :

(1) $f$ has maximal rank, and

(2) $\left.f_{*}\right|_{\left(\operatorname{Ker} f_{*}\right)} \perp$ is a linear isometry.

We say that $f$ is an almost Hermitian submersion if $f$ is a Riemannian submersion which, additionally, is :

(3) an almost complex mapping.

Vectors on $M$ which are in the kernel of $f_{*}$ are tangent to the fibres $\left(F_{y}=\right.$ $\left.f^{-1}(y), y \in B\right)$ and are called vertical vectors. Vectors which are orthogonal to the vertical distribution are said to be horizontal. We denote the vertical and horizontal distributions in the tangent bundle of the total space $M$ by $V(M)$ and $H(M)$, respectively. Then $T(M)$ enjoys an orthogonal decomposition: $T(M)=V(M) \oplus H(M)$. The orthogonal projection mappings are denoted $\mathscr{V}: T(M) \rightarrow V(M)$ and $\mathscr{H}: T(M) \rightarrow H(M)$ respectively. Since the fibres of a Riemannian submersion are closed, regularly embedded submanifolds [21] of the complete manifold $M$, the vertical distribution is completely integrable. In general, the horizontal distribution $H(M)$ is not completely integrable. Finally, we remark that the assumption of completeness for $M$ assures that $f: M \rightarrow B$ is a locally trivial Riemannian fibre space [11].

2.1. Proposition. Let $f: M \rightarrow B$ be an almost Hermitian submersion. Then the horizontal and vertical distributions determined by $f$ are $J$-invariant, i.e., $J\{V(M)\}=V(M)$ and $J\{H(M)\}=H(M)$. 
Proof. Let $W \in V(M)$. Since $f$ is almost complex, $J W$ is vertical. Now let $X \in H(M)$, and let $W$ be an arbitrary vertical vector. Then $g(J X, W)=$ $-g(X, J W)=0$ and therefore $J X \in H(M)$.

2.2. Proposition. Let $f: M \rightarrow B$ be an almost Hermitian submersion. Then the fibres $f$ are almost complexly embedded closed submanifolds of $M$ of dimension $2(m-n)$.

Proof. With the inherited almost complex structure from $M$, a fibre $F_{y}$ is almost complexly embedded because the vertical distribution is $J$-invariant and completely integrable.

When discussing almost Hermitian submanifolds, we denote tensors and operators on the fibre submanifolds (resp. the base manifold) by a caret * (resp. an apostrophe '). For instance, $\nabla, \hat{\nabla}, \nabla^{\prime}$ denote the Levi-Civita connections on $M, F_{y}$, and $B$ respectively.

A smooth vector field $X$ on $M$ is basic for the almost Hermitian submersion $f: M \rightarrow B$ if

(1) $X$ is horizontal, and

(2) $X$ is $f$-related to a vector field on $B$, which depends on $X$ and is denoted $X_{*}$.

Notice that "basic" does not refer to any basis. There is a one-to-one relationship between basic vector fields on $M$ and vectors fields on $B$.

2.3. Proposition. Let $f: M \rightarrow B$ be an almost Hermitian submersion, and let $X$ and $Y$ be basic vector fields on $M$. Then

(a) $g(X, Y)=h\left(X_{*}, Y_{*}\right) \circ f$

(b) $J X$ is the basic vector field associated to $J^{\prime} X_{*}$,

(c) $\mathscr{H} \nabla_{X} Y$ is the basic vector field associated to $\nabla_{X_{*}}^{\prime} Y_{*}$,

(d) $\mathscr{H}[X, Y]$ is the basic vector field associated to $\left[X_{*}, Y_{*}\right]$.

Proof. We show (b). Assertions (a), (c), and (d) are properties of a Riemannian submersion [15]. We know that $J X$ is horizontal from Proposition 2.1. Since the map $f$ is almost complex, $J^{\prime} X_{*}$ is $f$-related to $J X$.

We now want to examine the influence of a given structure defined on the total space $M$ (e.g., almost Kähler) on the determination of the corresponding structure on the fibre submanifolds and the base manifold. We shall see for most cases that a given property on $M$ is induced onto $F_{y}$ and onto $B$ by $f$.

2.4. Theorem. Let $f: M \rightarrow B$ be an almost Hermitian submersion. If
(a) quasi-Kähler,
(b) almost Kähler,
(a) quasi-Kähler,
(b) almost Kähler,
$M$ is (c) almost Tachibana, then $F_{y}$ is (c) almost Tachibana,
(d) Kähler,
(e) Hermitian,
(d) Kähler,
(e) Hermitian.

Proof. See Proposition 1.2.

2.5. Theorem. Let $f: M \rightarrow B$ be an almost Hermitian submersion. If 

(a) quasi-Kähler,
(b) almost Kähler,
(a) quasi-Kähler,
(b) almost Kähler,
$M$ is (c) almost Tachibana, then $B$ is
(c) almost Tachibana,
(d) Kähler,
(e) Hermitian,
(d) Kähler,
(e) Hermitian.

Proof. We first claim that $\Phi=f^{*} \Phi^{\prime}$ on basic vector fields. If $X$ and $Y$ are basic vector fields on $M$, and $X_{*}$ and $Y_{*}$ are their associated vector fields on $B$, then

$$
\Phi(X, Y)=g(X, J Y)=h\left(X_{*}, J^{\prime} Y_{*}\right) \circ f=f^{*} \Phi^{\prime}(X, Y)
$$

Since $f^{*}$ commutes with $d$ on differential forms, we also see that $d \Phi=f^{*}\left(d^{\prime} \Phi^{\prime}\right)$.

We begin the proof of Theorem 2.5 with assertion (b). If $M$ is almost Kähler, then clearly $f^{*} d^{\prime} \Phi^{\prime}=0$. But $f^{*}$ is a linear isometry because $f$ is a Riemannian submersion. So $d^{\prime} \Phi^{\prime}=0$ and therefore $B$ is almost Kähler. To establish assertion (a), it is now sufficient to remark that $f^{*}$ preserves the bidegree of differential forms.

Suppose that $M$ is almost Tachibana. It is easy to see that the basic vector field associated to $\nabla_{X_{*}}^{\prime} J\left(X_{*}\right)$ for any vector field $X_{*}$ on $B$ is $\mathscr{H} \nabla_{X} J(X)$ which vanishes on $M$. Thus (c) is shown. Similarly, the basic vector field on $M$ associated to the Nijenhuis tensor $N^{\prime}\left(X_{*}, Y_{*}\right)$ on $B$ is $\mathscr{H} N(X, Y)$. Therefore, when $M$ is Hermitian, $B$ is Hermitian. Finally, assertion (d) follows from (b) and (e).

In view of Theorems 2.4 and 2.5, an almost Hermitian submersion $f: M \rightarrow B$ for which both $M$ and $B$ are members of the class $\mathscr{P}$ in the lattice of almost Hermitian structures in Fig. 1 is called a $\mathscr{P}$-submersion. For instance, if $M$ is Kähler, then $f$ is a Kähler submersion.

Remark. A natural candidate for an almost Hermitian submersion is found in the construction of the only known class of almost Kähler (but not Kähler) manifolds. Tachibana and Okumura [17] and Yano and Ishihara [23] showed that the tangent bundle $T(M)$ of a Riemannian manifolds $M$ admits almost Kähler structures which are Kähler if and only if the manifold $M$ is locally flat. Bhatia and Prakash [2] constructed essentially the same structure on the cotangent bundle $T^{*}(M)$ of the Riemannian manifold $M$ and the same integrability theorem obtained. Now it is also known from the work of Gray [9], Dombrowski [3], and Sasaki [16] that the canonical bundle projection $\pi: T(M) \rightarrow M$ is a Riemannian submersion for certain choices of a Riemannian structure on $T(M)$. Therefore in the case when $M$ is almost Hermitian one may conjecture that $\pi: T(M) \rightarrow M$ (or $\pi: T^{*}(M) \rightarrow M$ ) is an almost Hermitian submersion and thus an almost Kähler submersion. Unfortunately, no matter how we combine the almost complex structures and Riemannian structures cited above, $\pi: T(M) \rightarrow M$ fails to be an almost Hermitian submersion (when $M$ is nonflat, of course) either because (a) $\pi$ is not an almost complex mapping with respect to the two almost complex structures, or (b) $\pi$ is not a 
Riemannian submersion with respect to the Riemannian structure which makes $T(M)$ into an almost Kähler space.

We turn now to an examination of the influence of the almost complex nature of the mapping $f$ on its configuration tensors. Let $E$ and $F$ be arbitrary vector fields on $M$. The $O^{\prime}$ Neill configuration tensors [15] of the Riemannian submersion $f: M \rightarrow B$ are given by:

$$
T_{E} F=\mathscr{H} \nabla_{\mathscr{V} E} \mathscr{V} F+\mathscr{V} \nabla_{\mathscr{V} E} \mathscr{H} F, \quad A_{E} F=\mathscr{V} \nabla_{\mathscr{H} E} \mathscr{H} F+\mathscr{H} \nabla_{\mathscr{H} E} \mathscr{V} F
$$

The properties of $T$ and $A$ are well-known, contained in O'Neill's original paper, and included here only for completeness.

2.6. Proposition. Let $f: M \rightarrow B$ be a Riemannian submersion. Then

(a) at any point $p \in M$, the linear operators $T_{E}$ and $A_{E}$ are skew-symmetric,

(b) $T_{E}\{H(M)\} \subseteq V(M)$ and $T_{E}\{V(M)\} \subseteq H(M)$,

(c) $A_{E}\{H(M)\} \subseteq V(M)$ and $A_{E}\{V(M)\} \subseteq H(M)$,

(d) $T$ is vertical and $A$ is horizontal, i.e., $T_{E}=T_{\gamma_{E}}$ and $A_{E}=A_{\mathscr{H E}}$,

(e) $T_{V} W=T_{W} V$ for all vertical vectors $V$ and $W$,

(f) $A_{X} Y=-A_{Y} X$ for all horizontal vectors $X$ and $Y$.

2.7. Proposition. Let $f: M \rightarrow B$ be a Riemannian submersion. Then

(a) for all $V, W \in V(M), T_{V} W$ is the configuration tensor $\hat{T}_{V} W$ of the immersed fibre submanifolds,

(b) $A$ vanishes if and only if the horizontal distribution is completely integrable,

(c) $T_{V} W=0$ for all $V, W \in V(M)$ if and only if $T_{V} V=0$ for all $V \in V(M)$,

(d) $T_{V}\{H(M)\}=0$ for all vertical $V$ if and only if $T_{V}\{V(M)\}=0$ for all vertical $V$ if and only if $T$ vanishes identically,

(e) $A_{X}\{H(M)\}=0$ for all horizontal $X$ if and only if $A_{X}\{V(M)\}=0$ for all horizontal $X$ if and only if $A$ vanishes identically.

Proof. Assertions (c), (d) and (e) were not in O'Neill's paper, but are simple exercises due to the skew-symmetry of $T$ and $A$.

2.8. Proposition. Let $f: M \rightarrow B$ be a Riemannian submersion, let $V$ and $W$ be vertical vector fields, and let $X$ and $Y$ be horizontal vector fields. Then
(a) $\nabla_{V} W=T_{V} W+\hat{\nabla}_{V} W$,
(b) $\nabla_{V} X=T_{V} X+\mathscr{H} \nabla_{V} X$,

(c) $A_{X} V=\mathscr{H} \nabla_{V} X$, whenever $X$ is basic,

(d) $\nabla_{X} V=A_{X} V+\mathscr{V} \nabla_{X} V$

(e) $\nabla_{X} Y=A_{X} Y+\mathscr{H} \nabla_{X} Y$.

Now we can begin to examine how the almost Hermitian structure on $M$ places restrictions on $T$ and $A$.

2.9. Theorem. Let $f: M \rightarrow B$ be a quasi-Kähler submersion, $V$ and $W$ vertical vectors, and $X$ and $Y$ horizontal vectors. Then
(a) $T_{V} J W=T_{J V} W$,
(b) $T_{J V} X=-J T_{V} X$,
(c) $A_{X} J X=0$,
(d) $A_{X} J Y=-A_{Y} J X$.

Proof. Assertion (a) follows from the similar result on the configuration tensor of the immersed fibre submanifolds. To see (b), note that

$$
g\left(T_{J V} X, W\right)=-g\left(T_{J V} W, X\right)=-g\left(T_{V} J W, X\right)=-g\left(J T_{V} X, W\right) .
$$


Then the nondegeneracy of $g$ implies $T_{J V} X=-J T_{V} X$. For a manifold which is quasi-Kähler, we have

$$
\nabla_{X} J X-\nabla_{J X} X=J \nabla_{X} X+J V_{J X} J X .
$$

Using the vertical part of this equation we find

$$
A_{X} J X-A_{J X} X=J A_{X} X+J A_{J X} J X=0 .
$$

Therefore $A_{X} J X=0$.

Assertion (d) follows from (c) by the standard polarization trick.

2.10. Theorem. Let $f: M \rightarrow B$ be an almost Tachibana submersion. Then, for all vertical vectors $V$ and $W$,

(a) $T_{V} J W=J T_{V} W$

(b) $T_{J V} W=J T_{V} W$,

(c) $T_{V} J X=J T_{V} X$, for all horizontal $X$.

Proof. Using the same polarization trick as before, for (a) and (b) it will be sufficient to show that $T_{V} J V=J T_{V} V$. By direct calculation we have

$$
\begin{aligned}
T_{V} J V & =\nabla_{V} J V-\hat{\nabla}_{V} J V \\
& =\nabla_{V}(J)(V)+J \nabla_{V} V-\hat{\nabla}_{V}(J)(V)-J \hat{\nabla}_{V} V \\
& =J\left\{\nabla_{V} V-\hat{\nabla}_{V} V\right\}=J T_{V} V .
\end{aligned}
$$

Assertion (c) is an easy calculation.

2.11. Theorem. Let $f: M \rightarrow B$ be a Kähler submersion. If $V$ is a vertical vector field and $E$ is any vector field, then

$$
T_{V} J E=J T_{V} E .
$$

Proof. Obvious.

\section{Almost semi-Kähler submersions}

As is seen in Proposition 2.4 and Theorem 2.5, the almost Hermitian structure on $M$ (e.g., quasi-Kähler) completely determines that structure on the fibres and the base manifold of the almost Hermitian submersion $f: M \rightarrow B$, provided that the structure on $M$ is at least quasi-Kähler. This transference is related to the fact that all almost complex submanifolds of quasi-Kähler manifolds are minimally immersed. It is not known, in general, whether the same property of minimal submanifolds holds for almost semi-Kähler spaces, although it most probably does not. (If an almost complex submanifold of an almost semi-Kähler manifold has codimension 2, then it is minimal). The transition from quasi-Kähler to almost semi-Kähler is a critical juncture in the lattice of almost Hermitian structures (see Fig. 1) as we see when we require the integrability of the almost complex structure. Quasi-Kähler goes to Kähler while almost semi-Kähler goes to semi-Kähler, a class different from the class of Kähler manifolds. The interrelation between the minimality of all almost complex 
submanifolds, the complexification to a Kähler space, and the transference of the almost Hermitian structure on the total space of the almost Hermitian submersion to the base manifold is evident in the main theorem of this section, an analogue of Theorem 2.5 for the case when $M$ is almost semi-Kähler.

3.1. Theorem. Let $f: M^{2 m} \rightarrow B^{2 n}$ be an almost Hermitian submersion with $M$, an almost semi-Kähler manifold. Then $B$ is almost semi-Kähler if and only if the fibres $F_{y}$ of $f$ are minimal submanifolds of $M$.

Proof. We show that the partial coderivative of the Kähler form of $M$ with respect to $F_{y}$ acting on a basic vector field $X$ is equal to the negative of the codifferential of the Kähler form of the base manifold $B$ acting on the associated $f$-related vector field $X_{*}$. That is, we show $\tilde{\delta} \Phi(X)=-\delta^{\prime} \Phi^{\prime}\left(X_{*}\right)$.

Let $\left\{E_{1}, \cdots, E_{m_{-n}}, J E_{1}, \cdots, J E_{m_{-n}}, F_{1}, \cdots, F_{n}, J F_{1}, \cdots, J F_{n}\right\}$ be a local $J-$ basis for the smooth vector fields on $M$ whose horizontal vector fields are basic (the word "basic" is used in two senses). Then

$$
0=\delta \Phi(X)=\tilde{\delta} \Phi(X)-\sum_{i=1}^{n} \nabla_{F_{i}} \Phi\left(F_{i}, X\right)+\nabla_{J F_{i}} \Phi\left(J F_{i}, X\right)
$$

Consider the first term in the summation :

$$
\begin{aligned}
\nabla_{F_{i}} \Phi\left(F_{i}, X\right) & =g\left(\nabla_{F_{i}}\left(J F_{i}\right), X\right)-g\left(J \nabla_{F_{i}} F_{i}, X\right) \\
& =g\left(\mathscr{H}_{F_{i}}\left(J F_{i}\right), X\right)-g\left(\mathscr{H}\left(J \nabla_{F_{i}} F_{i}\right), X\right) \\
& =h\left(\nabla_{F_{i *}}^{\prime}\left(J^{\prime} F_{i^{*}}\right), X_{*}\right) \circ f-h\left(J^{\prime}\left(\nabla_{F^{*}}^{\prime} F_{i^{*}}\right), X_{*}\right) \circ f \\
& =\nabla_{F^{*}}^{\prime} \Phi^{\prime}\left(F_{i^{*}}, X_{*}\right) .
\end{aligned}
$$

Similarly,

$$
\nabla_{J F_{i}} \Phi\left(J F_{i}, X\right)=\nabla_{J^{\prime} F_{i *}}^{\prime} \Phi^{\prime}\left(J^{\prime} F_{i *}, X_{*}\right)
$$

Therefore

$$
0=\tilde{\delta} \Phi(X)+\delta^{\prime} \Phi^{\prime}\left(X_{*}\right)
$$

The theorem then follows immediately from Theorem 1.4.

3.1.1. Corollary. Let $M$ be semi-Kähler, and $f: M \rightarrow B$ an almost Hermitian submersion. Then $B$ is semi-Kähler if and only if the fibres of $f$ are minimally immersed.

Proof. Combine Theorems 2.5(e) and 3.1.

Eells \& Sampson [4] studied harmonic maps $f: M \rightarrow N$ between complete Riemannian manifolds. A map $f$ is said to be harmonic if it minimizes a certain energy integral, or, equivalently, if it is in the kernel of a generalized Laplacian operator defined by them. For instance, an immersion is harmonic if and only if it is minimal. A Riemannian submersion is harmonic if and only if its fibres are minimally immersed. Eells \& Sampson, using the curvature content of the 
Laplacian operator, were able to prove several results which restrict the existence of harmonic mappings based of the Riemannian curvatures or Ricci curvatures of $M$ and $N$. These results obviously apply to almost Hermitian submersions as we formulate in

3.1.2. Corollary. Let $M$ be an almost semi-Kähler manifold, and $f: M$ $\rightarrow B$ an almost Hermitian submersion. Then $B$ is almost semi-Kähler if and only if $f$ is harmonic.

Remark. Eells and Sampson also proved a smoothness theorem for harmonic mappings. In particular, $C^{3}$ harmonic maps are $C^{\infty}$. All of $\S \S 2$ and 3 can be stated in terms of $C^{3}$ mappings, so that we see that $C^{3}$ almost semi-Kähler submersions are smooth.

Theorem 3.1 allows us to say a great deal about the submanifolds of the two manifolds of an almost Hermitian submersion for which both of the manifolds $M$ and $B$ are almost semi-Kähler. In fact, we gain information about the Riemannian submanifolds of $M$ and $B$ without requiring that they have even dimension or be almost complexly immersed. In his thesis, Escobales proved

3.2. Theorem. Let $f: M \rightarrow B$ be a Riemannian submersion with minimal fibres. Then a submanifold $N$ of the base space $B$ is minimal in $B$ if and only if $f^{-1}(N)$ is minimal in $M$.

Proof. [5].

Thus we have

3.3. Theorem. Let $f: M \rightarrow B$ be an almost semi-Kähler submersion. Then a Riemannian (although not necessarily almost Hermitian) submanifold $N$ of $B$ is minimally immersed in $B$ if and only if its lift $f^{-1}(N)$ is minimally immersed in $M$.

\section{Kähler submersions}

Since all holomorphically immersed submanifolds of Kähler (in fact, quasiKähler) manifolds are minimal, it is obvious that the fibres of a Kähler submersion are minimal. Moreover, we shall see that the horizontal distribution of a Kähler submersion is completely integrable. This obviously places severe restrictions on the existence of Kähler submersions.

4.1. Theorem. The horizontal distribution of a Kähler submersion is completely integrable.

Proof. Let $f: M \rightarrow B$ be a Kähler submersion, $X$ a basic horizontal vector field on $M, Y$ horizontal and $V$ vertical. Then

$$
\begin{aligned}
g\left(A_{J X} Y, V\right) & =g\left(A_{X} J Y, V\right)=-g\left(J Y, \mathscr{H} \nabla_{V} X\right) \\
& =g\left(Y, \mathscr{H} \nabla_{V} J X\right)=g\left(Y, A_{J X} V\right)=-g\left(A_{J X} Y, V\right) .
\end{aligned}
$$

Therefore $A_{J X} Y=0$. By Proposition 2.8(c), the horizontal integrability tensor $A$ vanishes. 
A smooth mapping $f: M \rightarrow N$ between smooth Riemannian manifolds is said to be totally geodesic if $f_{*}$ preserves parallel translation. Vilms [18] classified totally geodesic Riemannian submersions and showed that a Riemannian submersion $f: M \rightarrow B$ is totally geodesic if and only if both configuration tensors $T$ and $A$ vanish. He also proved that a Riemannian submersion from a complete manifold $M$ is totally geodesic if and only if it is a fibre bundle with flat connection and with the group of isometries of a fibre as structural group.

4.1.1. Corollary. A Kähler submersion with totally geodesic fibres is a totally geodesic mapping.

4.1.2. Corollary. Let $f: M \rightarrow B$ be a Kähler submersion with totally geodesic fibres. Then $f$ is a smooth fibre bundle with flat connection, and $M$ is a locally product manifold.

Proof. [18, Cor. 3.6, p. 79].

4.1.3. Corollary. Let $M$ be a simply connected Kähler manifold, and $f: M \rightarrow B$ a Kähler submersion with totally geodesic fibres. Then $M$ is a Kähler product space, and $f$ is a holomorphic product projection mapping.

\section{Curvature relations}

A Riemannian submersion $f: M \rightarrow B$ is Riemannian sectional curvature increasing on horizontal 2-planes [15]. Similarly, we have found that an almost Hermitian submersion is holomorphic sectional curvature increasing on horizontal holomorphic 2-planes (a holomorphic 2-plane is determined by the orthogonal nonzero vectors $X$ and $J X)$.

It seems surprising, however, that a quasi-Kähler submersion $f: M \rightarrow B$ preserves the holomorphic sectional curvature on horizontal vectors, and, moreover, is holomorphic bisectional curvature decreasing on pairs of nonzero horizontal vectors. Restricting further to Kähler submersions, we find that such a submersion is holomorphic bisectional curvature preserving on pairs of horizontal vectors. This is a reflection of the complete integrability of the horizontal distribution, which was demonstrated in $\S 4$.

After stating the well-known properties of the Riemannian sectional curvature $K$ for Riemannian submersions, we study the holomorphic sectional and bisectional curvatures for the almost Hermitian submersion $f: M \rightarrow B$. Even when a horizontal vector field $X$ is not basic, we shall denote the vector field $f_{*} X$ on $B$ by $X_{*}$.

5.1. Proposition. Let $f: M \rightarrow B$ be a Riemannian submersion. If $X$ and $Y$ are horizontal vectors, and $V$ and $W$ are vertical vectors, then

(i) $K(V \wedge W)=\hat{K}(V \wedge W)-\left\{g\left(T_{V} V, T_{W} W\right)-\left\|T_{V} W\right\|^{2}\right\} /\|V \wedge W\|^{2}$,

(ii) $K(X \wedge V)=\|X\|^{-2}\|V\|^{-2}\left\{g\left(\left(\nabla_{X} T\right)_{V} V, X\right)+\left\|A_{X} V\right\|^{2}-\left\|T_{V} X\right\|^{2}\right\}$,

(iii) $\quad K(X \wedge Y)=K^{\prime}\left(X_{*} \wedge Y_{*}\right)-3\left\|A_{X} Y\right\|^{2} /\|X \wedge Y\|^{2}$. 
Proof. See [15].

Let $(M, g, J)$ be an almost Hermitian manifold. The holomorphic bisectional curvature [6] is defined for any pair of nonzero vectors $E$ and $F$ on $M$ via

$$
B(E, F)=\|E\|^{-2}\|F\|^{-2} g(R(E, J E) F, J F) .
$$

The holomorphic sectional curvature [7] assigns to the nonzero vector $E$ the value

$$
H(E)=B(E, E) .
$$

Thus $H(E)$ is the sectional curvature of the 2-plane spanned by the othogonal vectors $E$ and $J E$.

5.2. Theorem. Let $f: M \rightarrow B$ be an almost Hermitian submersion. Let $X$ and $Y$ be horizontal, and $V$ and $W$ vertical. Then the holomorphic bisectional curvatures satisfy

(i) $\quad B(V, W)=\hat{B}(V, W)+\|V\|^{-2}\|W\|^{-2}\left\{g\left(T_{V} J W, T_{J V} W\right)\right.$

$$
\left.-g\left(T_{V} W, T_{J V} J W\right)\right\},
$$

(ii) $\quad B(X, V)=\|X\|^{-2}\|V\|^{-2}\left\{g\left(\left(V_{V} A\right)_{X} J X, J V\right)-g\left(A_{X} J V, A_{J X} V\right)\right.$

$$
\begin{aligned}
& +g\left(A_{X} V, A_{J X} J V\right)-g\left(\left(V_{J V} A\right)_{X} J X, V\right) \\
& \left.+g\left(T_{J V} X, T_{V} J X\right)-g\left(T_{V} X, T_{J V} J X\right)\right\},
\end{aligned}
$$

(iii) $\quad B(X, Y)=B^{\prime}\left(X_{*}, Y_{*}\right)-\|X\|^{-2}\|Y\|^{-2}\left\{2 g\left(A_{X} J X, A_{Y} J Y\right)\right.$

$$
\left.-g\left(A_{J X} Y, A_{X} J Y\right)-g\left(A_{Y} X, A_{J X} J Y\right)\right\} \text {. }
$$

Proof. (i) is just the Gauss equation for the fibre submanifolds. Relations (ii) and (iii) follow from certain equations of O'Neill concerning the covariant derivatives of the $T$ and $A$ tensors for a Riemannian submersion. Specifically, (ii) follows from equation $\left(2^{\prime}\right)$ and (iii) from equation (4) of [15].

5.3. Theorem. If $f: M \rightarrow B$ is a quasi-Kähler submersion, then

(i) $B(V, W)=\hat{B}(V, W)+\|V\|^{-2}\|W\|^{-2}\left\{\left\|T_{V} J W\right\|^{2}+\left\|T_{V} W\right\|^{2}\right\}$,

(ii) $\quad B(X, V)=\|X\|^{-2}\|V\|^{-2}\left\{g\left(\left(\nabla_{V} A\right)_{X} J X, J V\right)-g\left(A_{X} J V, A_{J X} V\right)\right.$

$$
\begin{aligned}
& +g\left(A_{X} V, A_{J X} J V\right)-g\left(\left(\nabla_{J V} A\right)_{X} J X, V\right) \\
& \left.-2 g\left(T_{V} X, T_{J V} J X\right)\right\},
\end{aligned}
$$

(iii) $B(X, Y)=B^{\prime}\left(X_{*}, Y_{*}\right)+\|X\|^{-2}\|Y\|^{-2}\left\{\left\|A_{X} J Y\right\|^{2}+\left\|A_{X} Y\right\|^{2}\right\}$.

Proof. A simple substitution of the various properties of the $T$ and $A$ tensors with respect to $J$ for the quasi-Kähler case as found in Theorem 2.9.

5.3.1. Corollary. If $f: M \rightarrow B$ is a quasi-Kähler submersion, then, for all vertical vectors $V$ and $W$, 


$$
B(V, W) \geq \hat{B}(V, W),
$$

and equality holds if and only if the fibres of $f$ are totally geodesic submanifolds of $M$.

5.3.2. Corollary. If $f: M \rightarrow B$ is a quasi-Kähler submersion, then, for all horizontal vectors $X$ and $Y$,

$$
B(X, Y) \geq B^{\prime}\left(X_{*}, Y_{*}\right),
$$

and equality holds if and only if the horizontal distribution is completely integrable.

5.4. Theorem. If $f: M \rightarrow B$ is an almost Tachibana submersion, then

(i) $\quad B(V, W)=\hat{B}(V, W)+2\|V\|^{-2}\|W\|^{-2}\left\|T_{V} W\right\|^{2}$,

(ii) $\quad B(X, V)=\|X\|^{-2}\|V\|^{-2}\left\{g\left(\left(\nabla_{V} A\right)_{X} J X, J V\right)-g\left(A_{X} J V, A_{J X} V\right)\right.$

$$
\begin{aligned}
& +g\left(A_{X} V, A_{J X} J V\right)-g\left(\left(V_{J V} A\right)_{X} J X, V\right) \\
& \left.+2\left\|T_{V} X\right\|^{2}\right\} .
\end{aligned}
$$

Proof. Assertion (i) is obvious from Theorems 2.10(a) and 5.3(i), and assertion (ii) follows from Theorems 2.10(c) and 5.3(iii).

5.5. Theorem. If $f: M \rightarrow B$ is a Kähler submersion, then

(i) $B(X, V)=2\|X\|^{-2}\|V\|^{-2}\left\|T_{V} X\right\|^{2}$ ，

(ii) $B(X, Y)=B^{\prime}\left(X_{*}, Y_{*}\right)$.

Proof. An immediate consequence of the previous theorem and Theorem 4.1.

5.5.1. Corollary. If $M$ is a Kähler manifold of constant holomorphic bisectional curvature $b$, and $f: M \rightarrow B$ is a Kähler submersion, then $B$ is a Kähler manifold of constant holomorphic bisectional curvature $b$.

5.5.2. Corollary. For Kähler submersions,

$$
B(X, V) \geq 0 \text {. }
$$

5.5.3. Corollary. If $M$ is a Kähler manifold with strictly negative holomorphic bisectional curvature, then there do not exist any Kähler submersions $f: M \rightarrow B$.

5.5.4. Corollary. If $M$ is a Kähler manifold with nonpositive holomorphic bisectional curvature, and $f: M \rightarrow B$ is a Kähler submersion, then $f$ is a totally geodesic mapping, and $M$ is a locally product mapping.

Proof. $B(E, F) \leq 0$ implies $B(X, V)=0$ for all horizontal $X$ and all vertical $V$. Therefore $T_{V} X=0$. Thus $T$ vanishes by Proposition 2.7(d), and Corollary 4.1.1 applies.

5.5.5. Corollary. The only Kähler submersions from a simply connected 
Kähler manifold $M$ of nonpositive holomorphic bisectional curvature are holomorphic product projection mappings.

We now adapt Theorems 5.2 through 5.5 to the holomorphic sectional curvature tensor.

5.6. Theorem. Let $f: M \rightarrow B$ be an almost Hermitian submersion, $X$ a horizontal vector, and $V$ a vertical vector. Then

(i) $H(V)=\hat{H}(V)+\|V\|^{-4}\left\{\left\|T_{V} J V\right\|^{2}-g\left(T_{V} V, T_{J V} J V\right)\right\}$,

(ii) $H(X)=H^{\prime}\left(X_{*}\right)-3\|X\|^{-4}\left\|A_{X} J X\right\|^{2}$.

Proof. The equations follow immediately from Theorem 5.2.

5.6.1. Corollary. For an almost Hermitian submersion $f: M \rightarrow B$,

$$
H(X) \leq H^{\prime}\left(X_{*}\right)
$$

5.7. Theorem. If $f: M \rightarrow B$ is a quasi-Kähler submersion, then

(i) $H(V)=\hat{H}(V)+\|V\|^{-4}\left\{\left\|T_{V} V\right\|^{2}+\left\|T_{V} J V\right\|^{2}\right\}$,

(ii) $H(X)=H^{\prime}\left(X_{*}\right)$.

Proof. (i) is an easy calculation due to the known properties of the $T$ tensor for quasi-Kähler spaces. Assertion (ii) follows from Theorem 2.9(c).

5.7.1. Corollary. In the case of a quasi-Kähler submersion,

$$
H(V) \geq \hat{H}(V)
$$

and equality holds if and only if the fibres of $f$ are totally geodesic.

Proof. The second assertion follows from Theorem 2.7(c).

5.7.2. Corollary. A quasi-Kähler submersion preserves the holomorphic sectional curvature on horizontal holomorphic 2-planes.

5.7.3. Corollary. Let $f: M \rightarrow B$ be a quasi-Kähler submersion, and suppose that $M$ is of constant holomorphic sectional curvature $c$. Then $B$ is a quasi-Kähler manifold of constant holomorphic sectional curvature $c$.

5.8. Theorem. Let $f: M \rightarrow B$ be an almost Tachibana submersion. Then

$$
H(V)=\hat{H}(V)+2\|V\|^{-4}\left\|T_{V} V\right\|^{2} .
$$

Proof. Use Theorem 2.10(a.)

\section{Cohomology}

In [19], the author announced the following theorem which motivated this study of almost Hermitian submersions.

6.1. Theorem. Let $f: M \rightarrow B$ be a Riemannian submersion between 
compact complete Riemannian manifolds. If the fibres of $f$ are minimal submanifolds of $M$, then

$$
b_{1}(B) \leq b_{1}(M) .
$$

Upon applying Theorem 3.1 we immediately obtain

6.2. Theorem. Let $f: M \rightarrow B$ be an almost Hermitian submersion between compact almost semi-Kähler manifolds. Then

$$
b_{1}(B) \leq b_{1}(M) \text {. }
$$

Although Theorem 6.2 does give us necessary conditions for the existence of an almost Hermitian submersion with only slight restrictions on the metric structures of the manifolds, it does not provide results of the type known for certain compact Kähler manifolds concerning $b_{p, q}$, the number of linearly independent harmonic forms of bidegree $(p, q)$. For instance, it is a classic result of Hodge theory that if $M$ is a compact Kähler manifold of constant positive holomorphic sectional curvature, then $b_{0,0}=b_{1,1}=\cdots=b_{m, m}=1$, and all the other $b_{p, q}$ are 0 .

The bidegree grading of the differential forms on an almost complex manifold has not been extensively documented, so we include a brief introduction here for completeness. Following Koszul [13], we decompose the complexified smooth vector fields on an almost complex manifold $\left(M^{2 m}, J\right)$ as

$$
\mathscr{D}(M) \otimes C=\mathscr{D}(M)^{+} \oplus \mathscr{D}(M)^{-},
$$

where $\mathscr{D}(M)^{+}=\{X \mid J X=i X\}$ and $\mathscr{D}(M)^{-}=\{X \mid J X=-i X\}$. Let $\Lambda^{r}(M)$ denote the complex differential $r$-forms on $M$. A differential form $\alpha \in \Lambda^{p+q}(M)$ is said to be of bidegree $(p, q)$ whenever more than $p$ of the $X_{i}$ being in $\mathscr{D}(M)^{-}$ or more than $q$ of the $X_{i}$ being in $\mathscr{D}(M)^{+}$force $\alpha\left(X_{1}, \cdots, X_{p+q}\right)=0$. We denote the space of differential $(p, q)$-forms on $(M, J)$ by $\Lambda^{p, q}(M)$.

It is well-known that the classical exterior differentiation operator $d$ takes a $(p, q)$-form to a sum of forms of various bidegrees [12], i.e.,

$$
d\left\{\Lambda^{p, q}\right\} \subseteq \Lambda^{p-1, q+2}+\Lambda^{p, q+1}+\Lambda^{p+1, q}+\Lambda^{p+2, q-1} .
$$

Suppose now that we have an almost Hermitian manifold $(M, g, J)$. The codifferential operator $\delta: \Lambda^{p+q} \rightarrow \Lambda^{p+q-1}$ defined on $M$ with respect to the Riemannian structure $g$ sends a $(p, q)$-form to a sum of complex differential forms of bidegrees $(p-2, q+1),(p-1, q),(p, q-1)$, and $(p+1, q-2)$. With these two facts, we can calculate the effect of the Laplacian operator $\Delta=-d \delta-\delta d$ on forms of bidegree $(p, q)$. In fact,

$$
\begin{aligned}
\Delta\left\{\Lambda^{p, q}\right\} \subseteq & \Lambda^{p-3, q+3}+\Lambda^{p-2, q+2}+\Lambda^{p-1, q+1} \\
& +\Lambda^{p, q}+\Lambda^{p+1, q-1}+\Lambda^{p+2, q-2}+\Lambda^{p+3, q-3} .
\end{aligned}
$$


We say that a differential form of bidegree $(p, q)$ is harmonic if it is harmonic as a $(p+q)$-form $(\Delta \alpha=0)$. We denote the complex vector space of harmonic $(p, q)$-forms on $M$ by $\mathscr{H}^{p, q}(M)$. The complex dimension of $\mathscr{H}^{p, q}(M)$ is denoted $b_{p, q}$.

One of the reasons why Hodge-Yano-Bochner theory works so well on Kähler manifolds is that the Laplacian operator is bidegree preserving on such manifolds. However, on a general almost Hermitian manifold $(M, g, J)$ the seven-part decomposition of $\Delta \alpha$ is probably too cumbersome to allow close analysis. In certain cases, we have been able to determine inequalities between the $b_{p, q}$ for the manifolds $M$ and $B$ of an almost Hermitian submersion $f: M \rightarrow B$.

We recall now the general idea of the proof of Theorem 6.1 . The classic Hodge theorem says that the vector space of real harmonic $r$-forms on an orientable compact Riemannian manifold $(M, g)$ is isomorphic to the classical de Rham cohomology space $H_{d}^{r}(M, R)$ and therefore to the classical real cohomology space $H^{r}(M, R)$ by de Rham's Theorem. Thus the number of linearly independent harmonic $r$-forms is exactly the $r$-th Betti number $b_{r}$. It is a trivial exercise to verify that on a compact orientable manifold an r-form $\alpha$ is harmonic if and only if $d \alpha=0$ and $\delta \alpha=0$ simultaneously. In a previous study [20], we classified those smooth manifold mappings $f: M \rightarrow N$ which satisfy $f^{*} \delta_{N}=\delta_{M} f^{*}$ on the differential $r$-forms of the Riemannian manifold $N$. It was found that a necessary condition is that $f: M \rightarrow N$ be a Riemannian submersion. Since $f^{*}$ always commutes with the $d$ operator, it follows that such a $\delta$-commuting manifold map takes harmonic $r$-forms on the compact orientable $N$ to harmonic $r$-forms on the compact orientable $M$. The fact that $f$ is a Riemannian submersion implies that $f^{*}$ is a linear isometry, and the inequality for Betti numbers in Theorem 6.1 is obtained. In the case, $r=1$, we found that a sufficient condition on the Riemannian submersion $f: M \rightarrow N$ which will ensure commutation with the codifferential is the minimality of the fibre submanifolds. Hence Theorem 6.1.

With this outline in mind, we wish to attack the same problem for the case of forms of bidegrees $(1,0)$ and $(0,1)$. The crucial fact which makes the analysis approachable is that $\delta\left\{\Lambda^{1,0}\right\}$ is a subspace of the single vector space $\Lambda^{0,0}=$ $C^{\infty}(M)$. Likewise for $\delta\left\{\Lambda^{0,1}\right\}$. Since the almost complex mapping $f$ preserves the bidegree of differential forms, the above argument gives

6.3. Theorem. Let $f: M \rightarrow B$ be an almost complex smooth manifold mapping between complete almost Hermitian manifolds. Then $f^{*}$ commutes with the codifferential operator $\delta$ on complex differential $(1,0)$-forms and $(0,1)$-forms if and only if $f: M \rightarrow B$ is an almost Hermitian submersion with minimally immersed fibres.

As we argued before, this leads immediately to

6.4. Theorem. Let $f: M \rightarrow B$ be an almost Hermitian submersion between compact almost Hermitian manifolds. If the fibres of $f$ are minimal, then 


$$
b_{1,0}(B)_{1} \leq b_{1,0}(M), \quad b_{0,1}(B) \leq b_{0,1}(M) .
$$

By combining Theorem 3.1 with the last theorem we obtain

6.5. Theorem. Let $M$ and $B$ be compact almost semi-Kähler manifolds. If there exists an almost Hermitian submersion $f: M \rightarrow B$, then

$$
b_{1,0}(B) \leq b_{1,0}(M), \quad b_{0,1}(B) \leq b_{0,1}(M) .
$$

\section{Almost quaternionic submersions}

An almost quaternionic manifold [7] is a quadruple $(M, I, J, K)$, where $I$, $J$ and $K$ are almost complex structures on the smooth manifold $M$ satisfying $K=I J=-J I$. A Riemannian almost quaternionic manifold $(M, g, I, J, K)$ is said to be q-almost Hermitian if it is almost Hermitian with respect to any two of the three almost complex structures $I, J$ and $K$. In exactly the same manner, we define q-almost semi-Kähler, q-quasi-Kähler, q-almost Kähler, q-almost Tachibana, q-Kähler, q-Hermitian and q-semi-Kähler manifolds. That is, when the Kähler forms of two of the three almost complex structures themselves satisfy the relevant definition given before Fig. 1 .

Let $(M, g, I, J, K)$ be a $q$ - $\mathscr{P}$-manifold where $\mathscr{P}$ is one of the classes in Fig. 1. Let $(S, h, \hat{I}, \hat{J}, \hat{K})$ be a $q$-almost Hermitian manifold which at the same time is a submanifold of $M$ and an almost Hermitian submanifold of $M$ with respect to each of the three almost complex structures $I, J$ and $K$. If $S$ is in the class $\mathscr{P}$ with respect to any two of the three almost complex structures $\hat{I}, \hat{J}$ and $\hat{K}$, then $S$ is called a $q-\mathscr{P}$-submanifold of $M$.

Gray [7] proved the following.

7.1. Lemma. A q-quasi-Kähler submanifold of a q-quasi-Kähler manifold is totally geodesically immersed.

A q-almost Hermitian submersion is a smooth mapping $f:(M, g, I, J, K)$ $\rightarrow\left(B, h, I^{\prime}, J^{\prime}, K^{\prime}\right)$ between $q$-almost Hermitian manifolds which is an almost Hermitian submersion with respect to each of the pairs of almost complex structures $\left(I, I^{\prime}\right),\left(J, J^{\prime}\right)$ and $\left(K, K^{\prime}\right)$. There are not many interesting $q$-quasiKähler submersions as the following theorem shows.

7.2. Theorem. A q-quasi-Kähler submersion is a totally geodesic mapping.

Proof. Let $(M, g, I, J, K)$ and $\left(B, h, I^{\prime}, J^{\prime}, K^{\prime}\right)$ be $q$-quasi-Kähler manifolds, and $f: M \rightarrow B$ a $q$-quasi-Kähler submersion. If $V$ and $W$ are vertical vectors, then Lemma 7.1 implies that $T_{V} W=0$. Thus $T$ vanishes by Proposition 2.7(d). Let $X$ and $Y$ be horizontal vectors. Then Theorem 2.9(d) gives

$$
A_{X} Y=-A_{J X} J Y, \quad A_{X} Y=-A_{I X} I Y .
$$

Similarly,

$$
A_{I X} I Y=-A_{I J X} I J X
$$


Therefore $A_{X} Y=0$, and $A$ vanishes by Proposition 2.7(e).

7.2.1. Corollary. A q-quasi-Kähler submersion $f: M \rightarrow B$ with $M$ simply connected is a product projection mapping.

Remark. The $q$-almost semi-Kähler analogue of Theorem 3.1 obviously obtains, word for word.

\title{
Bibliography
}

[1] M. Ako, Fibred spaces with almost complex structures, Kōdai Math. Sem. Rep. 24 (1972) 482-505.

[2] N. Bhatia \& N. Prakash, Almost complex and almost product structures in cotangent bundle, Tensor, N. S. 20 (1969) 315-319.

[3] P. Dombrowski, On the geometry of the tangent bundle, J. Reine Agnew. Math. 210 (1962) 73-88.

[ 4 ] J. Eells, Jr. \& J. H. Sampson, Harmonic mappings of Riemannian manifolds, Amer. J. Math. 86 (1964) 109-160.

[ 5 ] R. Escobales, The differential geometry of Riemannian submersions, Ph.D. thesis, Notre Dame, 1972.

[ 6 ] S. I. Goldberg \& S. Kobayashi, Holomorphic bisectional curvature, J. Differential Geometry 1 (1967) 225-233.

[7] A. Gray, Minimal varieties and almost Hermitian submanifolds, Michigan Math. J. 12 (1965) 273-287.

[8] - Some examples of almost Hermitian manifolds, Illinois J. Math. 10 (1966) 353-366.

[9] - Pseudo-Riemannian almost product manifolds and submersions, J. Math. Mech. 16 (1967) 715-738.

[10] - Nearly Kähler manifolds, J. Differential Geometry 4 (1970) 283-309.

[11] R. Hermann, A sufficient condition that a mapping of Riemannian manifolds be a fibre bundle, Proc. Amer. Math. Soc. 11 (1960) 236-242.

[12] S. Kobayashi \& K. Nomizu, Foundations of differential geometry, Vol. II, Interscience, New York, 1969.

[13] J. L. Koszul, Variétés Kählerienes, Notes, Saõ Paulo, 1957.

[14] S. Kotō, Some theorems on almost Kählerian spaces, J. Math. Soc. Japan 12 (1960) $422-433$.

[15] B. O'Neill, The fundamental equations of a submersion, Michigan Math. J. 13 (1966) 459-469.

[16] S. Sasaki, On the differential geometry of tangent bundles of Riemannian manifolds, I \& II, Tôhoku Math. J. 10 (1958) 338-354, 14 (1962) 146-155.

[17] S. Tachibana \& M. Okumura, On the almost complex structure of tangent bundles of Riemannian spaces, Tôhoku Math. J. 14 (1962) 156-161.

[18] J. Vilms, Totally geodesic maps, J. Differential Geometry, 4 (1970) 73-79.

[19] B. Watson, The first Betti numbers of certain locally trivial fibre spaces, Bull. Amer. Math. Soc. 78 (1972) 392-393.

[20] - - ס-commuting maps and Betti numbers, Tôhoku Math. J. 27 (1975) 137-152.

[21] J. Wolf, Differentiable fibre spaces and mappings compatible with Riemannian metrics, Michigan Math. J. 11 (1964) 65-70.

[22] J. Wolf \& A. Gray, Homogeneous spaces defined by Lie group automorphisms. I \& II, J. Differential Geometry 2 (1968) 77-114, 115-159.

[23] K. Yano \& S. Ishihara, Fibred Spaces with Invariant Riemannian metric, Kōdai Math. Sem. Reps. 19 (1967) 317-360.

\author{
NNIVERSITY OF ORIENTE \\ Cumaná, Venezuela \\ UNIVERSITY OF SiMON BOLlVAR \\ Sartenejas, Venezuela \\ Case Western Reserve University
}


